\title{
QU CARINAE: A SNeIa PROGENITOR?
}

\author{
S. KAFKA ${ }^{1,4,5}$, R. ANDERSON ${ }^{2,4}$, AND R. K. HONEYCUTT ${ }^{3}$ \\ ${ }^{1}$ Cerro Tololo Inter-American Observatory (CTIO), Casilla 603, La Serena, Chile; stella@ caltech.edu \\ ${ }^{2}$ McMaster University, Department of Physics \& Astronomy ABB-241, 1280 Main St. W. Hamilton, ON L8S 4M1, Canada \\ ${ }^{3}$ Astronomy Department, Indiana University, Swain Hall West, Bloomington, IN 47405, USA \\ Received 2007 December 25; accepted 2008 January 24; published 2008 March 13
}

\begin{abstract}
Optical spectra obtained in 2006-2007 of the nova-like cataclysmic variable QU Car are studied for radial velocities (RVs), line profiles, and line identifications. We are not able to confirm the reported $10.9 \mathrm{~h}$ orbital period from 1982, partly because our sampling is not ideal for this purpose and also, we suspect, because our RVs are distorted by line profile changes due to an erratic wind. P-Cygni profiles are found in several of the emission lines, including those of C IV. Carbon lines are abundant in the spectra, suggesting a carbon enrichment in the donor star. The presence of [O III] $5007 \AA$ and [N II] $6584 \AA$ is likely due to a diffuse nebula in the vicinity of the system. The wind signatures in the spectra and the presence of nebular lines are in agreement with the accretion wind evolution scenario that has been suggested to lead to SNeIa. We argue that QU Car is a member of the V Sge subclass of cataclysmic variables, and a possible SNeIa progenitor. It is shown that the recent light curve of QU Car has $\sim 1$ mag low states, similar to the light curve of V Sge, strengthening the connection of QU Car with V Sge stars, supersoft X-ray sources, and SNeIa progenitors.
\end{abstract}

Key words: novae, cataclysmic variables - stars: individual (QU Car) - stars: winds, outflows - supernovae: general

\section{INTRODUCTION}

Although identification of the progenitors of Supernovae Ia (SNeIa) remains controversial, it is accepted that they originate in binary systems in which at least one component is a white dwarf (WD); those systems are grouped under the wide umbrella of cataclysmic variables (CVs). CVs are semi-detached binaries in which a WD is accreting material from its lower main sequence or evolved companion (Warner 1995). When the magnetic field of the WD is low, a luminous accretion disk leads the gas onto the WD; if the magnetic field of the WD exceeds $\sim 10 \mathrm{MG}$, gas is guided onto the WD's magnetic poles via its magnetic field lines. Depending on the orbital period, the mass transfer rate $(\dot{M})$, the WD temperature, and the donor star's nature, CVs present a rich group of members, each providing valuable information on accretion physics, stellar evolution, binary star interaction, and cataclysmic explosions.

Current theories for SNeIa progenitors hold that, either via Roche lobe overflow of the companion or via a wind, the WD accumulates $\mathrm{H}$ - or He-rich material which is then burned to $\mathrm{C}$ and $\mathrm{O}$ on the WD. Under suitable conditions (determined primarily by the stability of $\dot{M}$ onto the WD) the WD mass reaches the Chandrasekhar limit initiating a series of thermonuclear reactions eventually leading to a SNeIa (Hillebrandt \& Niemeyer 2000). Although outlining this scenario is rather straightforward, the specifics are far from being understood or defined. For example, the nature of the companion star is uncertain, as well as how $\dot{M}$ remains low enough to prevent the expansion of the star's outer layers but high enough to allow stable H or He burning on the WD atmosphere without leading to a nova explosion. The WD mass necessary to initiate the thermonuclear reactions leading to the explosion is about $1.4 M_{\odot}$ and recent theoretical

\footnotetext{
4 Visiting Astronomer, Cerro Tololo Inter-American Observatory. CTIO is operated by AURA, Inc. under contract to the National Science Foundation. 5 Spitzer Science Center/Caltech, MS 220-6, 1200 E. California Blvd, Pasadena, CA 91125, USA.
}

models conclude that a fast rotating WD with initial mass as low as $0.7 M_{\odot}$ can accrete efficiently and eventually reach the Chandrasekhar limit (Hachisu et al. 2007). Currently, candidate progenitors are either double-degenerate (WD+WD) systems, or binaries that harbor a post-main-sequence companion (the single-degenerate binary, or SDB, scenario). Double-degenerate systems are less promising candidates due to their low overall mass or long orbital periods (Parthasarathy et al. 2007). Favorable candidates are the SDBs (Langer et al. 2000) for which stellar evolution codes predict the presence of substantial outflows; the outflows result from $\mathrm{H}$ - or He-rich material that is processed on the WD and expelled under appropriate conditions (e.g. Han \& Podsiadlowski 2004). Badenes et al. (2007) predict that the presence of stable, high-velocity winds $\left(v_{\text {outflow }} \geqslant 1000 \mathrm{~km} \mathrm{~s}^{-1}\right)$ should lead to the formation of cavities in the surrounding interstellar medium (ISM). However, such cavities are not observed around existing SNeIa remnants. Badenes et al. (2007) suggest that if outflows exist from SNeIa progenitors, they should be irregular and variable in time and should not be able to affect the surrounding pre-SN environment.

Among the most promising SNeIa progenitors are some types of transient supersoft X-ray sources (SSS), recurrent novae, and V Sge-type CVs. The latter is a small category of semi-detached binaries with high mass transfer rates $\left(\sim 10^{-7} M_{\odot} \mathrm{yr}^{-1}\right)$ allowing nuclear burning on the WD as mass transfer proceeds; these are considered to be the galactic counterparts of extragalactic SSS sources. Hachisu \& Kato (2003; hereafter HK03) model the long-term optical light curve of the prototype of the class, V Sge, and correlate the observed bright/faint states $(\sim 1$ mag in amplitude) of the system with the predictions of their accretion wind evolution (AWE) scenario. In short, the mass of the WD increases until it reaches a critical limit at which time the WD burns the accumulated $\mathrm{H}$ and expels a large portion of the processed material (primarily $\mathrm{C}$ and $\mathrm{O}$ ) in a fast wind. This wind sweeps away the disk surface increasing its luminosity (leading to an overall increase of the system brightness by up 


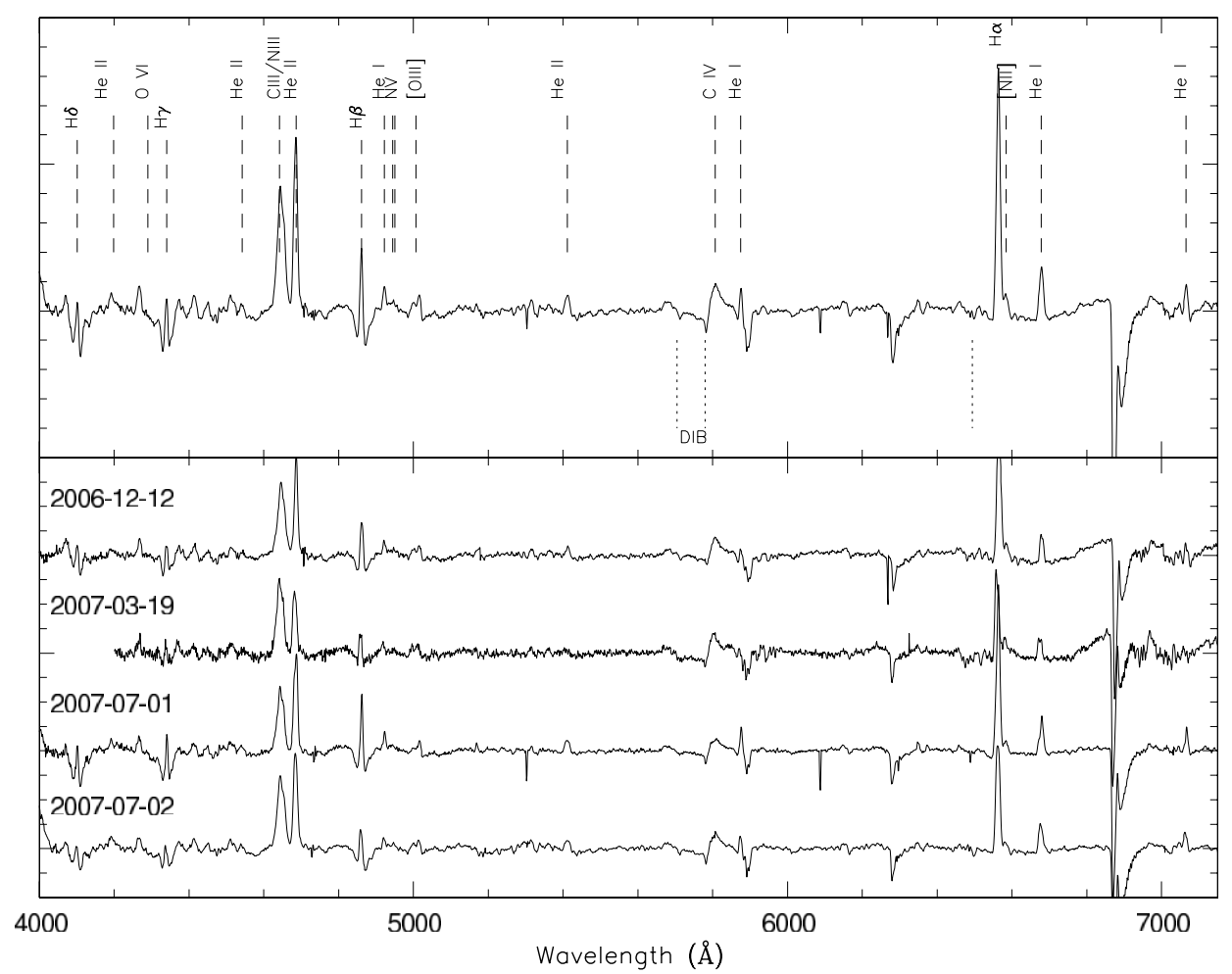

Figure 1. Examples of QU Car spectra from four epochs. The UT dates of observations are marked. The top spectrum is labeled with line identifications.

to $1 \mathrm{mag}$ ) and strips off the outer layers of the secondary star which becomes smaller than its Roche lobe; this halts mass transfer. The disk is then accreted onto the WD. Mass accretion eventually stops, leading to a cessation of the wind phase. Mass transfer starts again with the donor star regaining contact with its Roche lobe; this is also the beginning of a new cycle. At the same time, the WD accumulates enough material to eventually reach the Chandrasekhar limit and finally explode as a SNeIa.

In this paper we present a spectroscopic study of QU Car, an unusual yet bright and understudied CV. QU Car was first reported in Stephenson et al. (1968) as an irregular variable HD310376, similar to the bright low-mass X-ray binary Sco X-1 and the old nova HR Lyr. QU Car shares with Sco X-1 rapid and erratic photometric variations, as well as marked changes in the emission line spectrum. However, in contrast to Sco X-1, QU Car sometimes has He absorption features and relatively weak $\mathrm{X}$-ray emission (Schild 1969). In the optical, the system is noneclipsing and exhibits flickering of 0.1-0.2 mag on timescales of 1-10 min (Schild 1969; Gilliland \& Phillips 1982, hereafter GP82). In our study, we present new optical spectra suggesting that QU Car could be a member of the V Sge category and a likely SNeIa progenitor. The following section presents our data and data reduction technique, followed by the results of our analysis in Section 3 and our discussion in Section 4. We summarize our findings in Section 5.

\section{DATA ACQUISITION AND REDUCTION}

All our spectroscopic data were obtained with the RC Spectrograph on the CTIO 4-m Blanco telescope during four nights in 2006 and 2007. For each run, we used the KPGL3 grating, yielding a spectrum from $3800-7500 \AA$ at $1.2 \AA /$ pixel, providing a resolution of $\sim 3 \AA$. A log of our observations is
Table 1

Observing Log for QU Car

\begin{tabular}{lccc}
\hline \hline UT Date & \# Spectra & Exp. time (s) & Notes \\
\hline 2006 Dec 12 & 19 & 180 & Partly cloudy \\
2007 Mar 19 & 8 & 60 & Clear; dark \\
2007 Jul 01 & 2 & 180 & Clear; full moon \\
& 21 & 240 & \\
2007 Jul 02 & 8 & 300 & \\
\end{tabular}

provided in Table 1. For detector calibrations, we used the standard IRAF ${ }^{6}$ procedures, and for data reductions we used IRAF's onedspec/twodspec packages.

Due to passing clouds we had strong telluric features at 5920-6020 $\AA$ and 6447-6610 A for the 2006 December and 2007 March data. To correct for this absorption, we used data from the Wallace \& Livingston (2003) atlas and the IRAF task "telluric." Using "telluric" we interactively scaled the telluric features to match the observed spectra in regions having few stellar features; the goal was to minimize the rms difference in these regions. For all our spectra, we achieved a residual between the scaled correction spectrum and the spectrum of our star of $\lesssim 0.008$ (rms) for the selected regions. For the nights when we had passing clouds no standard stars were obtained; therefore the 2006 December and 2007 March data are not flux calibrated. For the 2007 June data we used LTT 4816 for flux calibration. The radial velocity (RV) corrected and summed spectrum of QU Car appears in Figure 1 (top) with

\footnotetext{
6 IRAF is distributed by the National Optical Astronomy Observatory, which is operated by the Association of Universities for Research in Astronomy, Inc., under cooperative agreement with the National Science Foundation.
} 
prominent emission/absorption features identified; summed individual spectra from each epoch of observations are also plotted.

\section{ANALYSIS}

\subsection{Radial Velocities}

We used IRAF/splot with a Gaussian fit to derive RVs and equivalent widths (EWs) of the emission lines. Conspicious lines include $\mathrm{H} \alpha, \mathrm{H} \beta$, the $\mathrm{C}$ III/N III/O II blend (4640-4650 $\AA$ ), and He II $4686 \AA$. The emission lines are rather weak with respect to the continuum. For example, the peak of the He II $4686 \AA$ emission line is typically only $3-5 \%$ above the continum. This He II line was used by GP82 to derive a binary orbital period of $10.9 \mathrm{~h}$, placing QU Car among the longer-period CVs. Table 2 lists our RV and EW measurements for this line. Our data do not cover a full orbit at any epoch; however measurements are available at various phases of different cycles. It was expected that our new data would be able to confirm the orbital period and provide a modern ephemeris, but that has not been the case. We first used a periodogram (Horne \& Baliunas 1986) on the GP82 He II data to confirm their period of $0.454 \pm 0.014$ days $(10.9 \mathrm{~h})$. Figure 2 compares the periodogram of the RVs from GP82 with a periodogram over the same frequency range for our new data, using both He II $4686 \AA$ and the central emission peak of $\mathrm{H} \alpha$. The location of the $10.9 \mathrm{~h}$ period adopted by GP82 is marked, and it is apparent that the periodograms of the two data sets are inconsistent. The GP82 data consist of 49 spectra on seven nights over 363 days. Our new data are comprised of 57 spectra on four nights over 202 days. The two data sets appear to be similar in time coverage, suggesting that the GP82 period may be in error. However, the GP82 data contain two continuous sequences of $7.8 \mathrm{~h}$ and $7.2 \mathrm{~h}$, while the longest sequences in our new data are $1.5 \mathrm{~h}$ and $1.6 \mathrm{~h}$; this difference appears to be critical for this system.

Figure 3 (top) shows the RV curve of the 1979 data from GP82, folded on the GP82 "combined He II" ephemeris. (The less extensive $1980 \mathrm{RVs}$ in GP82 are not plotted because we were not able to reproduce the GP82 phases for the 1980 data using their "combined He II" ephemeris.) The bottom panel shows our 2006-2007 He II RVs folded on the same ephemeris. Too many cycles have elapsed to expect the zero point of the GP82 ephemeris to remain valid for our new data; however, we expected some coherence when folded on the $10.9 \mathrm{~h}$ period. Instead we see only monotonic or nearly monotonic sequences which are more rapid than even the steepest part of the fitted curve in the top panel. The quoted $3 \%$ uncertainty on the $10.9 \mathrm{~h}$ period of GP82 accumulates to many cycles even over the eight month span of our data. Therefore the apparent sine-like curve between phases 0.3 and 0.7 in the bottom panel of Figure 3 is not physical, because the data on either side of phase 0.5 are separated by eight months. In fact, we carefully examined the power spectrum and folded RV curves for our data over the period range $0.05-10$ days, finding no periodicities of consequence.

It appears that either the period of QU Car is substantially shorter than the GP82 result, or that the intervals of rapid RV changes seen in our RV data are non-orbital in nature. More extensive measurements are needed to tell for sure, but we prefer the latter explanation given the current data. The reason is that the line profiles in QU Car are likely distorted on timescales of 1-3 h due to wind "events," similar to those described in optical wind lines for some nova-like CVs by Kafka et al.
Table 2

Radial Velocities and Equivalent Widths of the He II $4686 \AA$ A Line

\begin{tabular}{|c|c|c|}
\hline HJD & $\mathrm{RV}\left(\mathrm{km} \mathrm{s}^{-1}\right)$ & $\mathrm{EW}(\AA)$ \\
\hline $2,454,081.809$ & -136 & -1.9 \\
\hline $2,454,081.812$ & -115 & -2.0 \\
\hline $2,454,081.815$ & -151 & -2.0 \\
\hline $2,454,081.819$ & -156 & -2.2 \\
\hline $2,454,081.822$ & -182 & -1.9 \\
\hline $2,454,081.825$ & -199 & -1.8 \\
\hline $2,454,081.829$ & -203 & -1.8 \\
\hline $2,454,081.833$ & -223 & -1.9 \\
\hline $2,454,081.836$ & -217 & -1.9 \\
\hline $2,454,081.839$ & -216 & -2.0 \\
\hline $2,454,081.842$ & -217 & -2.1 \\
\hline $2,454,081.845$ & -226 & -2.1 \\
\hline $2,454,081.848$ & -224 & -2.1 \\
\hline $2,454,081.852$ & -235 & -2.3 \\
\hline $2,454,081.855$ & -222 & -2.3 \\
\hline $2,454,081.858$ & -218 & -2.3 \\
\hline $2,454,081.862$ & -197 & -2.5 \\
\hline $2,454,081.865$ & -176 & -2.5 \\
\hline $2,454,081.870$ & -235 & -2.4 \\
\hline $2,454,178.622$ & -288 & -1.5 \\
\hline $2,454,178.624$ & -266 & -1.5 \\
\hline $2,454,178.627$ & -226 & -1.5 \\
\hline $2,454,178.629$ & -225 & -1.4 \\
\hline $2,454,178.631$ & -237 & -1.9 \\
\hline $2,454,178.633$ & -257 & -1.8 \\
\hline $2,454,178.635$ & -256 & -1.6 \\
\hline $2,454,178.637$ & -282 & -1.8 \\
\hline $2,454,282.285$ & -96 & -2.6 \\
\hline $2,454,282.289$ & -111 & -2.6 \\
\hline $2,454,282.293$ & -93 & -2.6 \\
\hline $2,454,282.297$ & -43 & -2.8 \\
\hline $2,454,282.301$ & -9 & -2.9 \\
\hline $2,454,282.305$ & -14 & -2.8 \\
\hline $2,454,282.309$ & -20 & -2.8 \\
\hline $2,454,282.313$ & -32 & -2.7 \\
\hline $2,454,283.471$ & -55 & -2.5 \\
\hline $2,454,283.474$ & -68 & -2.6 \\
\hline $2,454,283.477$ & -75 & -2.5 \\
\hline $2,454,283.480$ & -36 & -2.5 \\
\hline $2,454,283.483$ & 12 & -2.5 \\
\hline $2,454,283.489$ & -7 & -2.4 \\
\hline $2,454,283.492$ & -23 & -2.6 \\
\hline $2,454,283.495$ & -26 & -2.5 \\
\hline $2,454,283.498$ & -38 & -2.7 \\
\hline $2,454,283.501$ & -41 & -2.3 \\
\hline $2,454,283.504$ & -35 & -2.6 \\
\hline $2,454,283.507$ & -73 & -2.6 \\
\hline $2,454,283.510$ & -93 & -2.5 \\
\hline $2,454,283.514$ & -100 & -2.5 \\
\hline $2,454,283.517$ & -106 & -2.9 \\
\hline $2,454,283.520$ & -107 & -3.0 \\
\hline $2,454,283.523$ & -128 & -2.7 \\
\hline $2,454,283.526$ & -129 & -3.0 \\
\hline $2,454,283.529$ & -120 & -2.8 \\
\hline $2,454,283.532$ & -129 & -2.7 \\
\hline $2,454,283.535$ & -134 & -2.9 \\
\hline $2,454,283.538$ & -177 & -2.9 \\
\hline
\end{tabular}

(2003) and Kafka \& Honeycutt (2004). Evidence supporting this conclusion includes the following: (1) QU Car clearly has an erratic wind (from the P Cyg profiles), (2) the He II 4686 line can have a strong wind component in some high-inclination NL CVs (Honeycutt et al. 1986), and (3) the GP82 data also display occasional nearly monotonic changes in RV over 


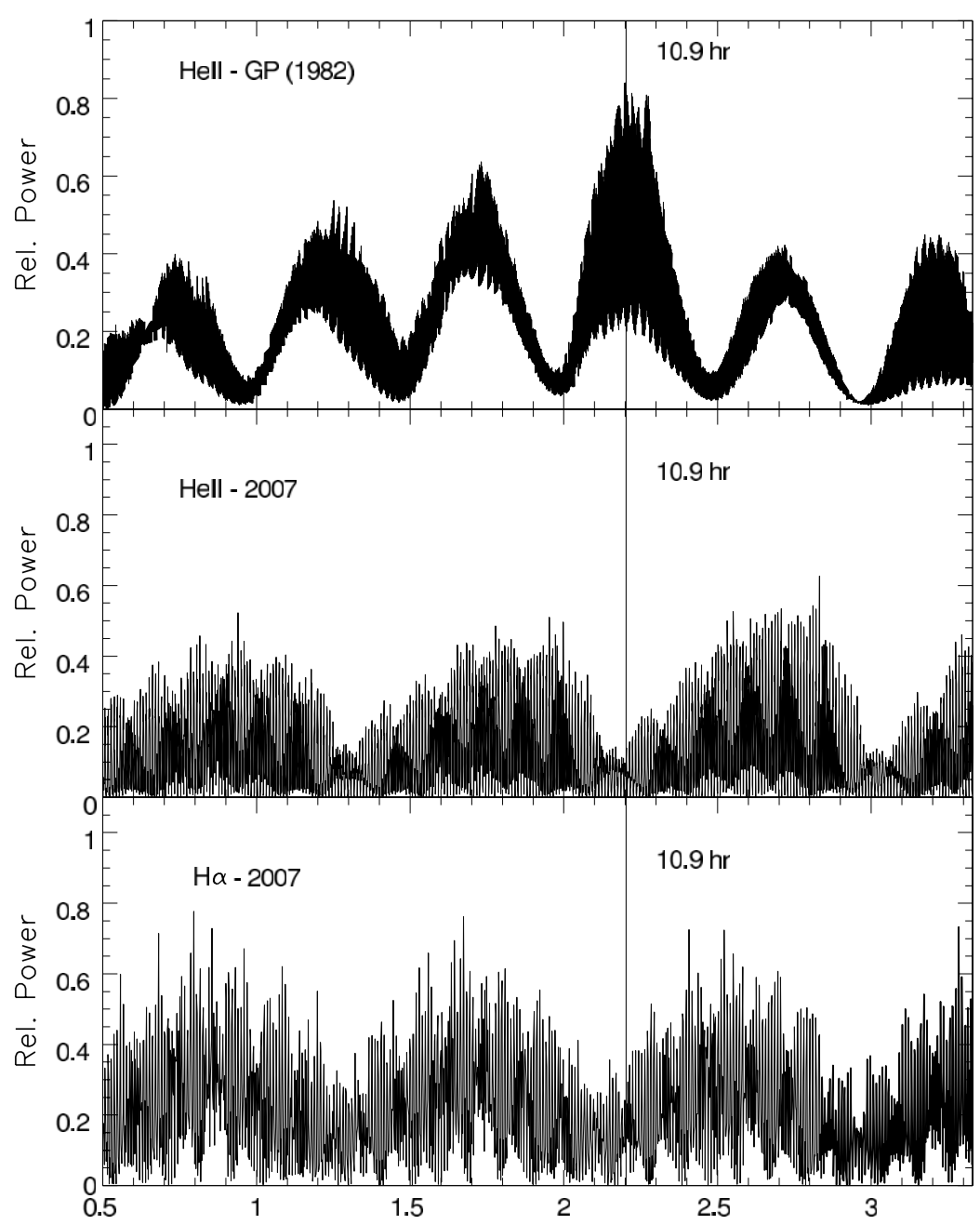

Figure 2. Periodogram of the GP82 He II data (top), our He II data (middle), and our H $\alpha$ data (bottom). The horizontal axis is frequency in days ${ }^{-1}$. The orbital period of $10.9 \mathrm{~h}$ from GP82 is marked in all plots.

1-3 $\mathrm{h}$ that are considerably faster than any portion of the fitted RV curve (one of these can be seen as the filled circles in the top panel of Figure 3). Such changes have little effect on the period analysis of GP82 because the lengths of their spectral sequences are considerably longer. This is not the case with our new data, making it impossible to improve or confirm the GP82 period study. A new comprehensive RV study of QU Car would certainly be helpful considering the importance (and brightness) of the system.

We also measured the RVs of the other emission lines; however none indicated any periodicity nor did they show any coherent variation with the GP82 orbital period. Schild (1969) and GP82 examined their short-term photometric data for periodic modulation in the $V$-band light curve, but found none. The $\sim 0.2$ mag "flaring" events appeared to be erratic on timescales of a few minutes. We examined the AAVSO light curve of the system (Figure 4) for periodicities but were unable to find any, even when we grouped the data to reduce the scatter.

\subsection{The Outflow from $Q U$ Car and Nebula Lines}

In high $\dot{M}$ disk CVs, winds often produce blueshifted absorption components to the emission lines. These P-Cygni profiles, most obvious in UV resonance lines such as C IV $1449 \AA$ and Si IV $1397 \AA$, are seen at times in both nova-like CVs and in dwarf novae during outburst. P-Cygni profiles appear mostly in low-inclination (i.e. disk almost face-on) CVs, indicating a bi-polar nature of the outflow. The lines are thought to form by resonance scattering in an accelerating wind, with velocities reaching $5000 \mathrm{~km} \mathrm{~s}^{-1}$. Although the origin of the wind is uncertain, it is generally thought to arise from the inner accretion disk/boundary layer. Some CVs are known to have reliable P-Cygni profiles in optical He I and/or H lines; e.g. BZ Cam (Ringwald \& Naylor 1998) and Q Cyg (Kafka et al. 2003). Those P-Cygni profiles are most conspicuous in the He I triplet lines at $5876 \AA$ and $7065 \AA$ whereas they are absent in the He I singlet line at $6678 \AA$ (despite the fact that these three lines have very similar excitation levels). Kafka et al. (2003) argued that this behavior is due to the strongly metastable $2^{3} \mathrm{~S}$ effective ground state of the triplets, which becomes overpopulated in conditions of low density and a dilute radiation field, overpopulating in turn the $2^{3} \mathrm{P}$ common lower level of He I $5876 \AA$ and $7065 \AA$, leading to absorption in a low density wind.

In QU Car, variable and erratic P-Cygni profiles in $\mathrm{N} \mathrm{v}$ $1238.8 \AA / 1242.8 \AA$ (doublet), O v $1371 \AA$ and Si IV $1398 \AA$ and C IV $1549 \AA$ have already been reported (Knigge et al. 

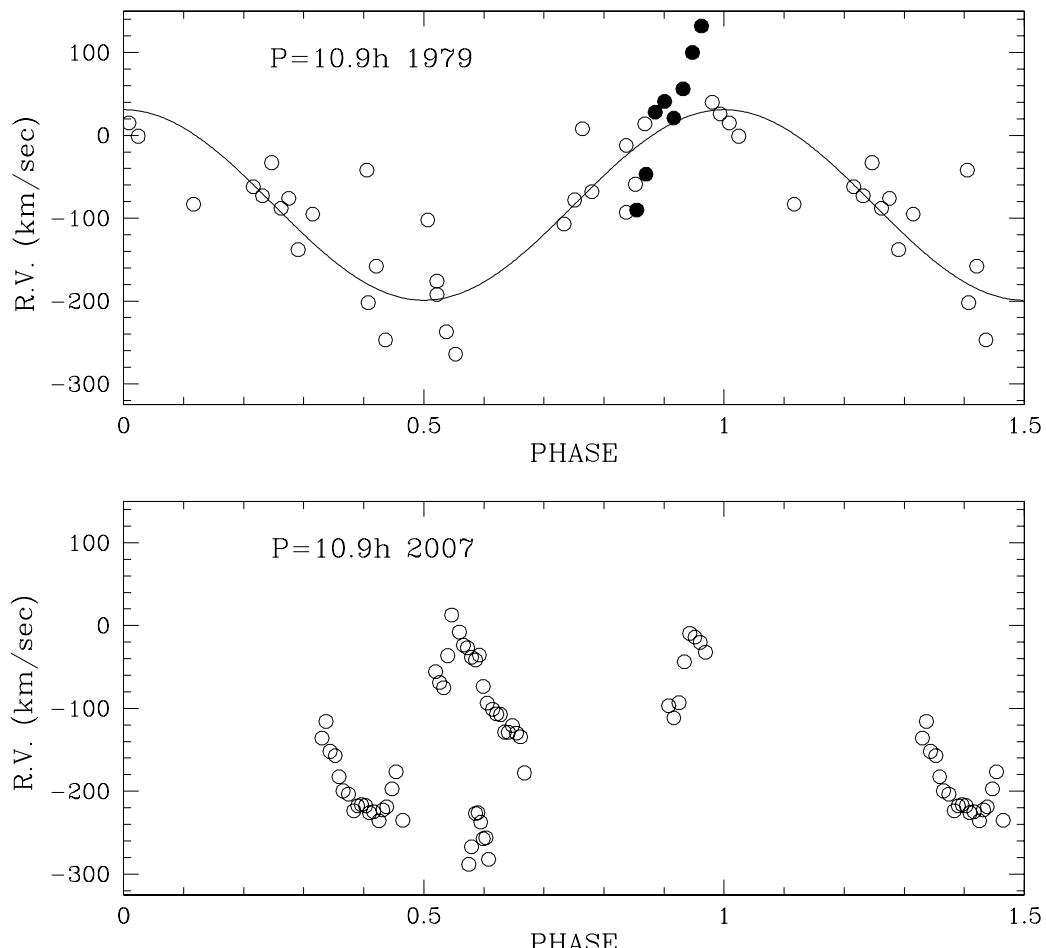

Figure 3. Folded RV curves of the He II 4686 line in QU Car. Top: 1979 data from GP82, along with their fitted curve. The solid points mark a continuous sequence in time having a rapid nearly monotonic RV change. Bottom: 2006-2007 data from this study, showing numerous rapid monotonic changes similar to the sequence marked in the top panel. See the text for details.
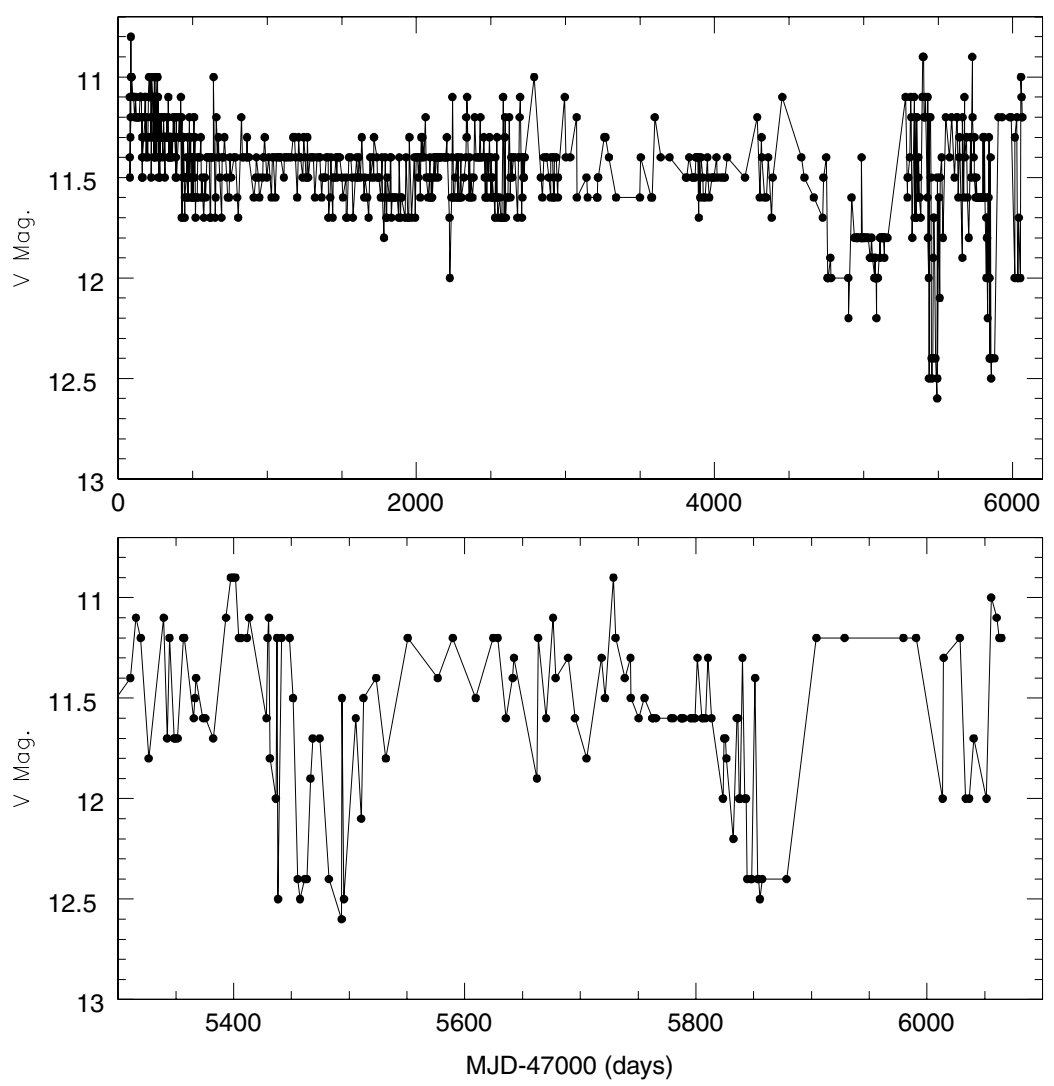

Figure 4. Top: 20 years of AAVSO light curve of QU Car. The system started showing V Sge-like bright/faint states (bottom) in mid-2002 (MJD 52430). 
Table 3

Equivalent Widths ${ }^{\mathrm{a}}$ of the Emission Lines

\begin{tabular}{|c|c|c|c|c|c|}
\hline Line $^{b}$ & Central wavelength & 2006 Dec 12 & 2007 Mar 19 & 2007 Jul 01 & $2007 \mathrm{Jul} 02$ \\
\hline $\mathrm{H} \delta$ & 4101 & -0.42 & $\cdots$ & -3.18 & -1.54 \\
\hline He II & 4199 & -0.37 & $\ldots$ & -0.15 & -0.25 \\
\hline Si II & 4265 & -0.39 & -0.46 & -0.32 & -0.26 \\
\hline $\mathrm{H} \gamma$ & 4340 & -1.58 & -0.20 & -2.11 & -0.24 \\
\hline He II & 4542 & -0.12 & -0.22 & -0.32 & -0.26 \\
\hline $\mathrm{C}$ III/N III/O II & 4642 & -3.09 & -3.08 & -3.05 & -3.56 \\
\hline He II & 4686 & -2.00 & -1.59 & -2.75 & -2.79 \\
\hline $\mathrm{H} \beta$ & 4861 & -13.94 & -0.58 & -3.17 & -7.60 \\
\hline He I & 4922 & -0.21 & -0.19 & -0.29 & -0.23 \\
\hline [O III] (full) & 5007 & -0.48 & -0.46 & -0.48 & -0.51 \\
\hline [O III] (blue) & & -0.31 & -0.19 & -1.16 & -0.45 \\
\hline [O III] (red) & & -0.28 & -0.30 & -0.24 & -0.28 \\
\hline He II & 5409 & -0.31 & $\ldots$ & -0.50 & -0.45 \\
\hline C III & 5696 & -0.60 & -0.32 & -0.31 & -0.21 \\
\hline C IV & 5801,5812 & -1.17 & -1.38 & -0.64 & -0.92 \\
\hline $\mathrm{C}_{\mathrm{IV}} \mathrm{abs}^{\mathrm{c}}$ & & 1.95 & 2.78 & 1.78 & 2.20 \\
\hline He I & 5875 & -0.12 & -0.13 & -0.31 & -0.20 \\
\hline $\mathrm{H} \alpha$ & 6563 & -3.78 & -2.26 & -4.23 & -3.29 \\
\hline$[\mathrm{N}$ II $]$ & 6584 & -0.71 & -0.57 & -0.32 & -0.27 \\
\hline He I & 6678 & -0.67 & -0.58 & -0.85 & -0.79 \\
\hline He I & 7065 & -0.33 & -0.17 & -0.51 & -0.51 \\
\hline
\end{tabular}

Notes.

a We follow the convention that the EW of emission lines are negative.

${ }^{b}$ Only the EW of the Balmer line emission cores are measured.

c These measurements are uncertain, since they overlap with the DIBs at $5780 \AA$ and $5705 \AA$.

Their inclusion here is to demonstrate the variation of the wind absorption between epochs of observations.

1994; Hartley et al. 2002; Drew et al. 2003). Their UV PCygni profiles have no apparent phase dependence and unknown time evolution. The modest velocities of the outflow (up to $\sim 2000 \mathrm{~km} \mathrm{~s}^{-1}$ ) suggested an outer-disk origin for the wind and an unusually high $\dot{M}$ for the system (Hartley et al. 2002). In our (and older) optical data the He I triplet lines in QU Car did not exhibit P-Cygni profiles (see Figure 1). It is likely that strong C IV emission at $5802 \AA$ overlaps any blueshifted absorption in the He I $5876 \AA$ line. Occasional shallow absorption dips appear in the blue side of the $\mathrm{H} \alpha$ line, reaching $\sim 1000 \mathrm{~km} \mathrm{~s}^{-1}$. At the same time a broad P-Cygni profile is present at the blue end of the C IV $5807 \AA$ line. The presence of diffuse interstellar bands at $5780 \AA$, $5705 \AA$, and $6494 \AA$ complicates measuring the strength of this blue-shifted absorption. However, one can discern relative changes in the strength of the absorption profiles between epochs. The EW of the absorption component of the $\mathrm{C}$ IV line is included in Table 3, indicating up to $50 \%$ variability in the strength of the wind at different epochs. This $\mathrm{C}$ IV line likely originates from a $\mathrm{C} \mathrm{v} \rightarrow \mathrm{C}$ IV recombination cascade requiring $490 \mathrm{eV}$, indicating the presence of a strong ionization field in the system. Such high ionization levels might also explain the lack of P-Cygni profiles in the lower-ionization He I lines. The velocity of the wind in $\mathrm{C}$ IV reaches $\sim 5700 \mathrm{~km} \mathrm{~s}^{-1}$; this is a lower limit since the C III $5696 \AA$ line overlaps the P-Cygni profile at its blue end.

We were surprised to find traces of [O III] in the spectrum of QU Car. Of the two components of the doublet, only the $5007 \AA$ component is present. This forbidden line appears in planetary nebula and active galactic nuclei (AGNs) as a result of photoionized gas around a central source (e.g. Dimitrijević et al. 2007). For AGNs the flux ratio in the doublet is near 3 (Dimitrijević et al. 2007). If the process governing [O III] emission in QU Car is similar to that in AGNs, then the [O III] $4959 \AA$ component is likely absent in QU Car due to its relative faintness. The detection of forbidden lines indicates the presence of a nebula. The line is weak and somewhat difficult to measure, but it appears that the [O III] line in QU Car has substantial RV variations, from $\sim 250$ (epoch 3 ) to $\sim-128 \mathrm{~km} \mathrm{~s}^{-1}$ (epoch 2).

In our QU Car spectra the [O III] $5007 \AA$ line has two components with nearly constant displacement from the line center of -500 and $370 \mathrm{~km} \mathrm{~s}^{-1}$. These likely arise from the front and back sides of an expanding shell or wind. We measured the EWs of the two components using Gaussian fits; although the red component hardly changes, the blue component appears to vary in strength (see Table 3). This line is weak, but we nevertheless judge these changes to be real. Similar behavior of [O III $5007 \AA$ is seen in some symbiotic stars, with the line being split into two components, and variability in strength over intervals of months (Kenyon 1986).

The presence in QU Car of [N II] $6584 \AA$ emission, which is also common in planetary nebulae, confirms the existence of a nebula in the vicinity of the star. Our data show EW variations in this line (more than $2 \times$ variation between epoch 1 and epoch 4), but again the line is weak. Undetected is [Si II] (at $6717 \AA$ and $6731 \AA$ ), which is characteristic of the nebulosity associated with the strong-wind CV BZ Cam (Greiner et al. 2001), and visual inspection of the spectra did not indicate any extended nebulosity. Overall, the presence of forbidden lines in QU Car is consistent with the outflows associated with the AWE mechanism, which we argue is operating in this system to produce the high-state/low-state features in the light curve (see Section 4), and the wind features in the spectrum. 

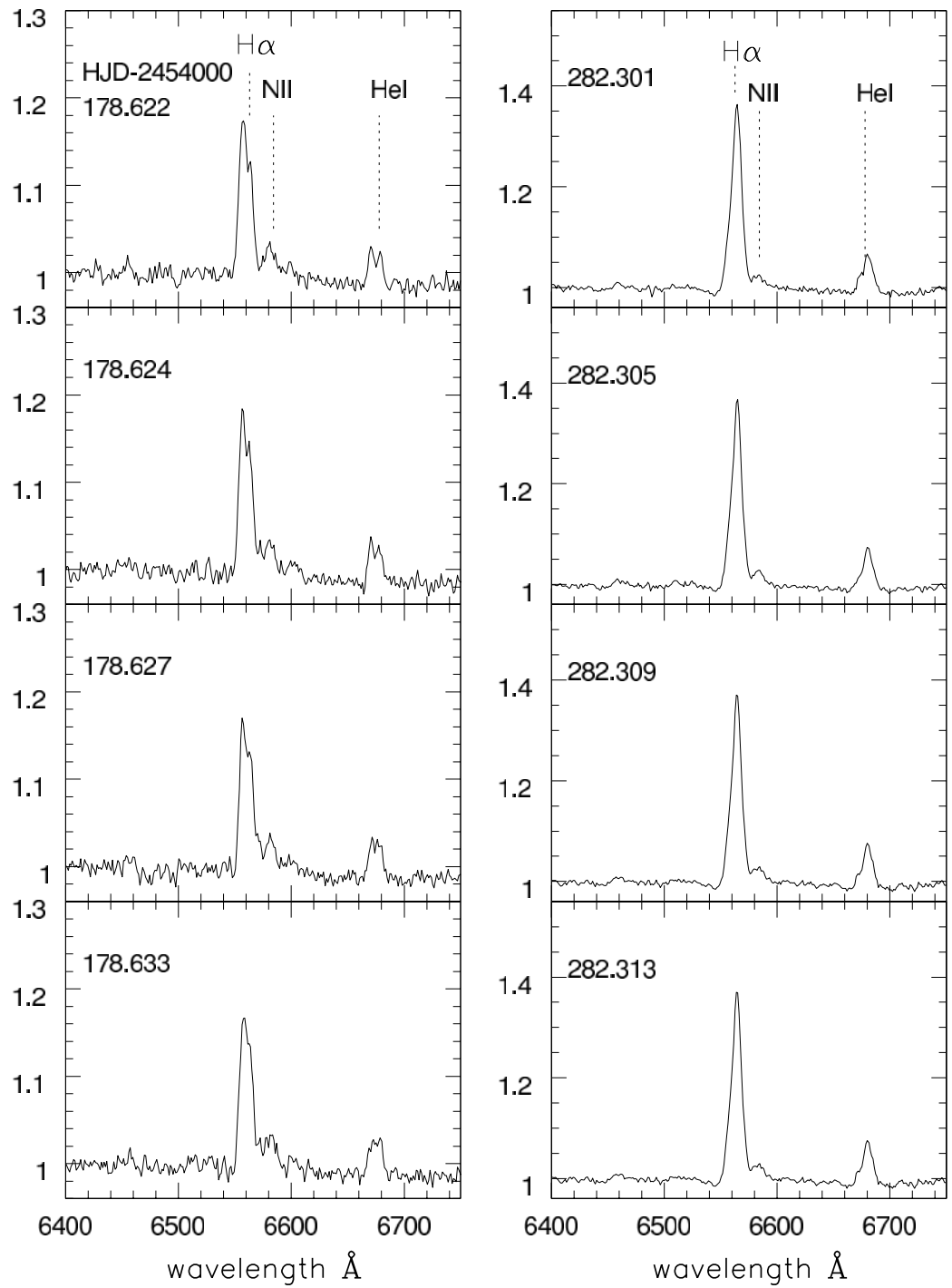

Figure 5. Examples of changes in the strength and profile of $\mathrm{H} \alpha$ and He I. The vertical scale is in units of the continuum and HJD-2,454,000 is shown on top of each plot. Note the structure in both $\mathrm{H} \alpha$ and $\mathrm{He}$ I on the left set of panels, and the lack of structure three months later (right panel). Note also that the lines in the right panels are twice the EW of those on the left. Finally, there is a weak P-Cygni profiles at times when the lines are strong (right panels).

\subsection{Hydrogen and Helium Emission Lines}

Figure 5 demonstrates graphically the variability in the strength and profile of hydrogen and helium lines in QU Car. Tables 2 and 3 also document the changes in EW. In the spectroscopic study of GP82, the $\mathrm{H} \beta$ emission line seems to be transient, disappearing between phases 0.2 and 0.7 , with a maximum EW of $\sim 3 \AA$. When present, the emission component of the line is superposed on a wide absorption trough. The phasing of the RV variations of the $\mathrm{H} \beta$ emission trailed the He II RV curve by $\mathrm{P} / 4$. Drew et al. (2003) searched for absorption features in the optical that could reveal the nature of the donor star. They discuss the presence of the He II $5411 \AA$ line and the complete absence of O VI $5290 \AA$, both of which are characteristic of SSSs. Both GP82 and Drew et al. (2003) measure the He II $4686 \AA / \mathrm{H} \beta$ EW ratio to be greater than 2 , in agreement with the typical value in SSSs. In our data, this ratio is always less than 1. Furthermore, in our data, the $\mathrm{H} \beta$ line has the largest $\mathrm{EW}$ values and $\mathrm{EW}$ variations ever reported in QU Car: the EW varies from $14 \AA$ to $0.6 \AA$ at two successive epochs. (Table 3 ). At the same time, the surrounding Balmer lines do not follow this variation. We remain puzzled as to how this situation can occur. The $\mathrm{H} \alpha$ line is mostly single peaked; however at times the second peak appears to be present (Figure 5). The He I $6678 \AA$ A, $5876 \AA$, and $7065 \AA$ lines seem to also have an additional (red) component at times.

Drew et al. (2003) described an overabundance of carbon in QU Car with respect to both helium and oxygen, suggesting that it is likely due to an R-type carbon donor star. A number of $\mathrm{C}$ emission lines are identified in Figure 1; however there is no evidence of any absorption features from the secondary star. Perhaps the ultra-luminous accretion disk of QU Car masks detection of the secondary. However, the presence of both C III $5696 \AA$ and C IV 5802, $5812 \AA$ is typical of carbon WolfRayet (W-R) stars and carbon-type central remnants of planetary nebulae, suggesting that the donor star may be carbon rich. Considering a wide range of possibilities, the strong carbon features could be due to (1) carbon generated by nuclear burning 
on the WD as part of the AWE cycle, (2) carbon-rich gas accreted onto the WD from a carbon-rich companion, or (3) a carbon W-R secondary star contributing directly to the optical light in QU Car. If the latter is the case, then (following the Crowther et al. (1998) classification criteria for W-R/WC stars), the ratio of C III $5696 \AA$ to C IV 5802, $5812 \AA$ implies WC7 for the QU Car secondary star. This assumes that C III $5696 \AA$ is not greatly affected by the absorption component of C IV.

\section{A V Sge STAR AND SNeIa PROGENITOR?}

Drew et al. (2003) used interstellar absorption features to derive a lower distance limit of $\sim 2 \mathrm{kpc}$ to QU Car, leading to $\dot{M} \sim 10^{-7} M_{\odot} \mathrm{y}^{-1}$, consistent with high $\dot{M}$ in V Sge stars. The members of this class are considered to have evolved donor stars that transfer gas with $\dot{M} \sim 10^{-7} M_{\odot} \mathrm{y}^{-1}$. Synthetic UV SEDs and an empirical mass-period relation lead to a w.d. mass of $\sim 1.2 M_{\odot}$ (Linnell et al. 2008), higher than the typical CV w.d. mass of $\sim 0.7 M_{\odot}$. The spectrum of QU Car seems similar to that of WX Cen, a member of the V Sge category and also a suggested SNeIA progenitor (Oliveira \& Steiner 2004). Overall, QU Car appears to possess the key properties for being a SNeIa progenitor candidate under the AWE mechanism: very high $\dot{M}$, erratic winds (plus nebular lines), and high/low states (as described below). Figure 4 shows the long-term AAVSO ${ }^{7}$ light curve of QU Car. Beginning in 2002 QU Car displayed 1 mag faint states resembling the faint states of the prototype of the class, V Sge (Robertson et al. 1997). In V Sge this repeated high/low state cycle has been interpreted as evidence for the accretion wind evolution scenario, leading to SNeIa (HK03). The AAVSO light curve for QU Car shows that such behavior is new for this system. We may be witnessing the onset of a V Sge (or SSS) phase in a CV.

\section{SUMMARY}

We have presented a new spectroscopic study of the bright nova-like QU Car. The highlights of this work are as follows:

1. We explored the orbital period of the system using He II RVs from this study and from GP82. We are unable to confirm the $10.9 \mathrm{~h}$ period of GP82. We argue that this situation is lilely due to rapid variations (compared to orbital) in the line profiles (P-Cygni) of the He II line due to an erratic wind, combined with our relatively short time series. We use as support for this argument the fact that the wind variability timescales in NL CVs are in this range, and the presence of fast monotonic RV changes in our data as well as in GP82.

2. Forbidden lines are detected in the QU Car spectrum, consistent with substantial mass outflow. The [O III] 5007 appears split, likely due to contributions from the front and back sides of an expanding flow.

3. It is pointed out that the light curve of QU Car has low states very similar to those in V Sge. In V Sge these low states are taken as evidence for the operation of accretion wind evolution cycle that is thought to lead to SNeIa. We propose that QU Car is also a strong candidate for a SNeIa progenitor, based on this and other similarities to V Sge systems. These similarites include high $\dot{M}$ and evidence for a strong wind from P-Cygni profiles and nebular lines.

It is surprising that so little optical work has been done on this bright, important system. It is important to determine/refine the orbital period of QU Car. Photometric monitoring is needed to define the characteristics of the photometric cycle and allow accurate comparisons with the predictions of the accretion wind model. Higher-resolution spectral data will provide information on the various ionization regions and possible emission lines from the nebula. High-resolution coronagraphic imaging should be employed to search for nebulosity. QU Car appears to be the brightest known member of the V Sge category, and a likely SNeIa progenitor. Further study can provide important information on the nature and the conditions that lead to mass accumulation onto the WD during the wind accretion cycle.

We gratefully acknowledge observations from the AAVSO International Database contributed by observers worldwide and used in this research. We would also like to thank our anonymous referee for a careful review of the manuscript.

\section{REFERENCES}

Badenes, C., Hughes, J. P., Bravo, E., \& Langer, N. 2007, ApJ, 662, 472 Crowther, P. A., De Marco, O., \& Barlow, M. J. 1998, MNRAS, 296, 367 Dimitrijević, M. S., Popović, L. Č., Kovačević, J., Dačić, M., \& Ilić, D. 2007, MNRAS, 374, 1181

Drew, J. E., Hartley, L. E., Long, K. S., \& van der Walt, J. 2003, MNRAS, 338,401

Gilliland, R. L., \& Phillips, M. M. 1982, ApJ, 261, 617 (GP82)

Greiner, J., et al. 2001, A\&A, 376, 1031

Hachisu, I., \& Kato, M. 2003, ApJ, 598, 527 (HK03)

Hachisu, I., Kato, M., \& Nomoto, K. 2007, arXiv:0710.0319

Han, Z., \& Podsiadlowski, P. 2004, MNRAS, 350, 1301

Hartley, L. E., Drew, J. E., \& Long, K. S. 2002, MNRAS, 336, 808

Hillebrandt, W., \& Niemeyer, J. C. 2000, ARA\&A, 38, 191

Horne, J. H., \& Baliunas, S. L. 1986, ApJ, 302, 757

Honeycutt, R. K., Schlegel, E. M., \& Kaitchuck, R. H. 1986, ApJ, 302, 388

Kafka, S., \& Honeycutt, R. K. 2004, AJ, 128, 2420

Kafka, S., Tappert, C., Honeycutt, R. K., \& Bianchini, A. 2003, AJ, 126, 1472 Kenyon, S. J. 1986, The Symbiotic Stars (New York: Cambridge Univ. Press)

Knigge, C., Drew, J. E., Hoare, M. G., \& La Dous, C. 1994, MNRAS, 269, 891 Langer, N., Deutschmann, A., Wellstein, S., \& Houmlflich, P. 2000, A\&A, 362, 1046

Linnell, A. P., Godon, P., Hubeny, I., Sion, E. M., Szkody, P., \& Barrett, P. E. 2008, arXiv:0801.0704

Oliveira, A. S., \& Steiner, J. E. 2004, MNRAS, 351, 685

Parthasarathy, M., Branch, D., Jeffery, D. J., \& Baron, E. 2007, New Astron. Rev., 51,524

Ringwald, F. A., \& Naylor, T. 1998, AJ, 115, 286

Robertson, J. W., Honeycutt, R. K., \& Pier, J. R. 1997, AJ, 113, 787

Schild, R. E. 1969, ApJ, 157, 709

Stephenson, C. B., Sanduleak, N., \& Schild, R. E. 1968, ApJ, 1, L247

Wallace, L., \& Livingston, W. 2003, An Atlas of the Solar Spectrum in the Infrared from 1850 to $9000 \mathrm{~cm}^{-1}$ (1.1 to $\left.5.4 \mu \mathrm{m}\right)$, revised by L. Wallace and W. Livingston, NSO Technical Report, 2003 (Tucson: National Solar Observatory, National Optical Astronomy Observatory)

Warner, B. 1995, Cataclysmic Variable Stars, Cambridge Astrophysics Series (Cambridge: Cambridge Univ. Press) 